PLASMA ELECTRODE POCKELS CELL SUBSYSTEM PERFORMANCE IN THE NATIONAL IGNITION FACILITY

F. Barbosa, P.A. Arnold, A.F. Hinz, R.A. Zacharias, C.W. Ollis, E.S. Fulkerson, B. Mchale, A. Runtal, C.L. Bishop

August 2, 2007

IEEE Pulsed Power Conference Albuquerque, NM, United States June 21, 2007 through June 22, 2007 
This document was prepared as an account of work sponsored by an agency of the United States Government. Neither the United States Government nor the University of California nor any of their employees, makes any warranty, express or implied, or assumes any legal liability or responsibility for the accuracy, completeness, or usefulness of any information, apparatus, product, or process disclosed, or represents that its use would not infringe privately owned rights. Reference herein to any specific commercial product, process, or service by trade name, trademark, manufacturer, or otherwise, does not necessarily constitute or imply its endorsement, recommendation, or favoring by the United States Government or the University of California. The views and opinions of authors expressed herein do not necessarily state or reflect those of the United States Government or the University of California, and shall not be used for advertising or product endorsement purposes.

This work was performed under the auspices of the U. S. Department of Energy by University of California, Lawrence Livermore National Laboratory under Contract W-7405-Eng-48. 


\title{
PLASMA ELECTRODE POCKELS CELL SUBSYSTEM PERFORMANCE IN THE NATIONAL IGNITION FACILITY
}

\author{
F. Barbosa, P.A Arnold, A.F. Hinz, R.A. Zacharias, C.W. Ollis, E.S. Fulkerson, B. Mchale, A. \\ Runtal \\ Lawrence Livermore National Laboratory \\ 7000 East Avenue, Livermore CA 94551
}

\author{
C.L. Bishop \\ IAP World Services \\ 7000 East Avenue, Livermore CA 94551
}

\begin{abstract}
The Plasma Electrode Pockels Cell (PEPC) subsystem is a key component of the National Ignition Facility, enabling the laser to employ an efficient four-pass main amplifier architecture. PEPC relies on a pulsed power technology to initiate and maintain plasma within the cells and to provide the necessary high voltage bias to the cells' nonlinear crystals. Ultimately, nearly 300 high-voltage, high-current pulse generators will be deployed in the NIF in support of PEPC.

Production of solid-state plasma pulse generators and thyratron-switched pulse generators is now complete, with the majority of the hardware deployed in the facility. An entire cluster (one-fourth of a complete NIF) has been commissioned and is operating on a routine basis, supporting laser shot operations. Another cluster has been deployed, awaiting final commissioning. Activation and commissioning of new hardware continues to progress in parallel, driving toward a goal of completing the PEPC subsystem in late 2007.
\end{abstract}

\section{INTRODUCTION}

The National Ignition Facility (NIF) is a $1.8 \mathrm{MJ}$, 192beam laser fusion driver with a goal of achieving scientific breakeven - with a fusion yield that equals (and eventually exceeds) laser input to the target. The Plasma Electrode Pockels Cell (PEPC) is a key technology within $\mathrm{NIF}$, working in conjunction with a thin-film polarizer to create an electro-optical switch in the main amplifier cavity. This optical switch permits the optical pulses to be trapped and then released, allowing the NIF to take advantage of a four-pass architecture in the main amplifier, thus reducing costs and minimizing the required size of the facility while maximizing performance.

The PEPC system has successfully been deployed in the NIF facility as a fully functional system. Currently, well over half of the PEPC line replaceable units (LRUs), including their high voltage, low voltage, gas and vacuum supporting equipment have been installed in NIF. In the NIF a line replaceable unit is a device that is removed to a separate facility for repair; fully operational units are "plugged in" for defective units to effect rapid replacement and minimum downtime.

\section{SUBSYSTEM OVERVIEW}

PEPC is one of the more complex line replaceable units in the National Ignition Facility in terms of design and operation. The following sections introduce the details of operation of the PEPC during a laser shot.

\section{A. PEPC Operation in the Main Amplifier Cavity}

NIF employs a multi-pass amplifier architecture that requires a mechanism for switching the beam in and out of the main amplifier cavity. PEPC, working in conjunction with the main amplifier polarizer, functions as an optical switch for the main amplifier cavity. This enables NIF to take advantage of the gain and attendant cost-savings afforded by a four-pass design. The beamline depicted in Figure 1 represents but one of the 192 beams that will eventually become operational in NIF.

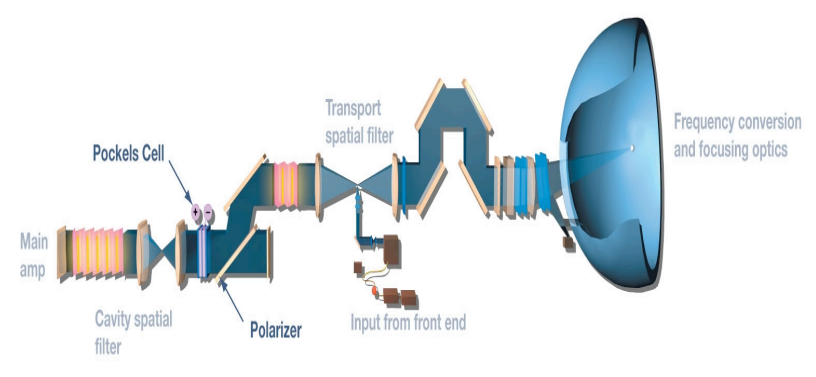

Figure 1. Location of the PEPC in the NIF Laser System. 
Like all Pockels cells, the PEPC takes advantage of electric field-induced birefringence, a characteristic of certain non-linear crystals including the crystal of choice in $\mathrm{NIF}, \mathrm{KH}_{2} \mathrm{PO}_{4}$ (KDP). However, the required lateral dimensions of the KDP, the square cross-section of the NIF beam (nominal aperture of $40 \mathrm{~cm} \mathrm{x} 40 \mathrm{~cm}$ ), optical power and energy densities preclude the possibility of using more conventional techniques (such as ring electrodes or transparent, conductive thin films) for impressing a uniform electric field on the KDP across the entire face of the crystal. Thus, a plasma electrode is formed in the low-pressure ( $\sim 70$ millitorr) process gas that resides on both sides of an array of four crystals in each NIF Pockels cell. An electron density of $\sim 10^{12} \mathrm{~cm}^{-3}$ provides sufficient conductivity to produce an equipotential surface, thereby allowing a uniform electric field to be applied to the entire KDP crystal. [1,2] The electric field induces changes to the refractive index of the crystals, which causes the $90^{\circ}$ rotation in the polarization of the laser light on two of the four passes through the laser cavity.

\section{B. Step-by-step Operation of the Plasma Electrode Pockels Cell}

A detailed sequence of switching events within the main laser cavity (for a single beam) is as follows: prior to a laser shot, the Pockels cell lies in a quiescent state. The regions on either side of the crystal are evacuated to below $1 \times 10^{-5}$ Torr by an on-board turbo molecular pump. Minutes before the shot, a $\mathrm{He}-\mathrm{O}_{2}$ gas mixture is flowed into and through the cell, raising the pressure to 70 millitorr in the gas cells on both sides of the crystal. [3]

Approximately 300 milliseconds before the arrival of the laser beam in the cavity, low voltage, low current power supplies in the PEPC Plasma Pulse Generators (PPGs) apply an excitation to the low pressure $\mathrm{He} / \mathrm{O}_{2}$ mixture. The excitation voltage initially exceeds $1 \mathrm{kV}$ but drops quickly to a steady state value of $300 \mathrm{~V}$. This has the effect of initiating and then maintaining very low conductivity plasma.

Ten microseconds before the beam arrives, the electron density of the plasma is raised to approximately $10^{12} \mathrm{~cm}^{-3}$ by applying a multi-kiloampere current resulting from the discharge of a high voltage capacitor in the PPG. The plasma forms near-equipotential surfaces on both sides of the crystal to which a pulse can be applied and via which a uniform electric field can be impressed on the crystal. By necessity, the plasma is transparent to the laser beam traversing the cavity.

Meanwhile, the linearly polarized optical pulse propagates to the cavity polarizer. The s-plane polarization of the beam is such that the light reflects off of the polarizer and into the Pockels cell. The cell is still "off" at this point (that is, no electric field is applied to the KDP. As noted, the plasma has already been created on both sides of crystal). The beam crosses the cell essentially unperturbed (except for non-zero absorption of the crystal and small but non-zero reflections at each optical surface) and continues on its path to the far end of the amplifier, and thus completing Pass 1. Reflecting off LM1, the beam begins Pass 2.

The Switch Pulse Generators (SPG) are purposely triggered to arrive 100ns after the laser beam has cleared the Pockels cell during pass 1 . The Pockels cell is now in its "on" state, the applied voltage is such that it induces birefringence in the crystal, which effectively rotates the polarization of the beam by $90^{\circ}$. In particular, the amplified laser beam, having made its second pass through the amplifier laser cavity, now passes through the active cell and, as described, the polarization rotates. The beam is now p-plane polarized with respect to the polarizer and the beam passes through the polarizer, then reflects off LM2, and passes through the polarizer once more; the Pockels cell is still in the "on" state as the beam begins Pass 3. The polarization of the light is rotated again by $90^{\circ}$, returning it to the original state (spolarization) of the first pass through the amplifier.

The beam crosses the main amplifier two more times (Passes 3 and 4) and returns yet again to the Pockels cell. By this time, the applied voltage has dropped to zero and the cell is in an "off" state. Thus, the beam passes through the Pockels cell with its polarization unaltered. The splane polarized light reflects off the polarizer and back into the 2-pass power amplifier.

The electrical requirements for the Plasma Electrode Pockels Cell can be summarized as follows: Initiate low density plasma within the individual channels of the cell. Raise the electron density in the channels to a level sufficient to provide equipotential surfaces for the application of the required crystal bias. Apply crystal bias during passes 2 and 3 , effecting a $90^{\circ}$ polarization on each of the passes. Avoid any beam interactions on passes 1 and 4. [3] Figure 2 illustrates the step-by-step interaction between the Plasma Electrode Pockels Cell and one of NIF's laser beams. 

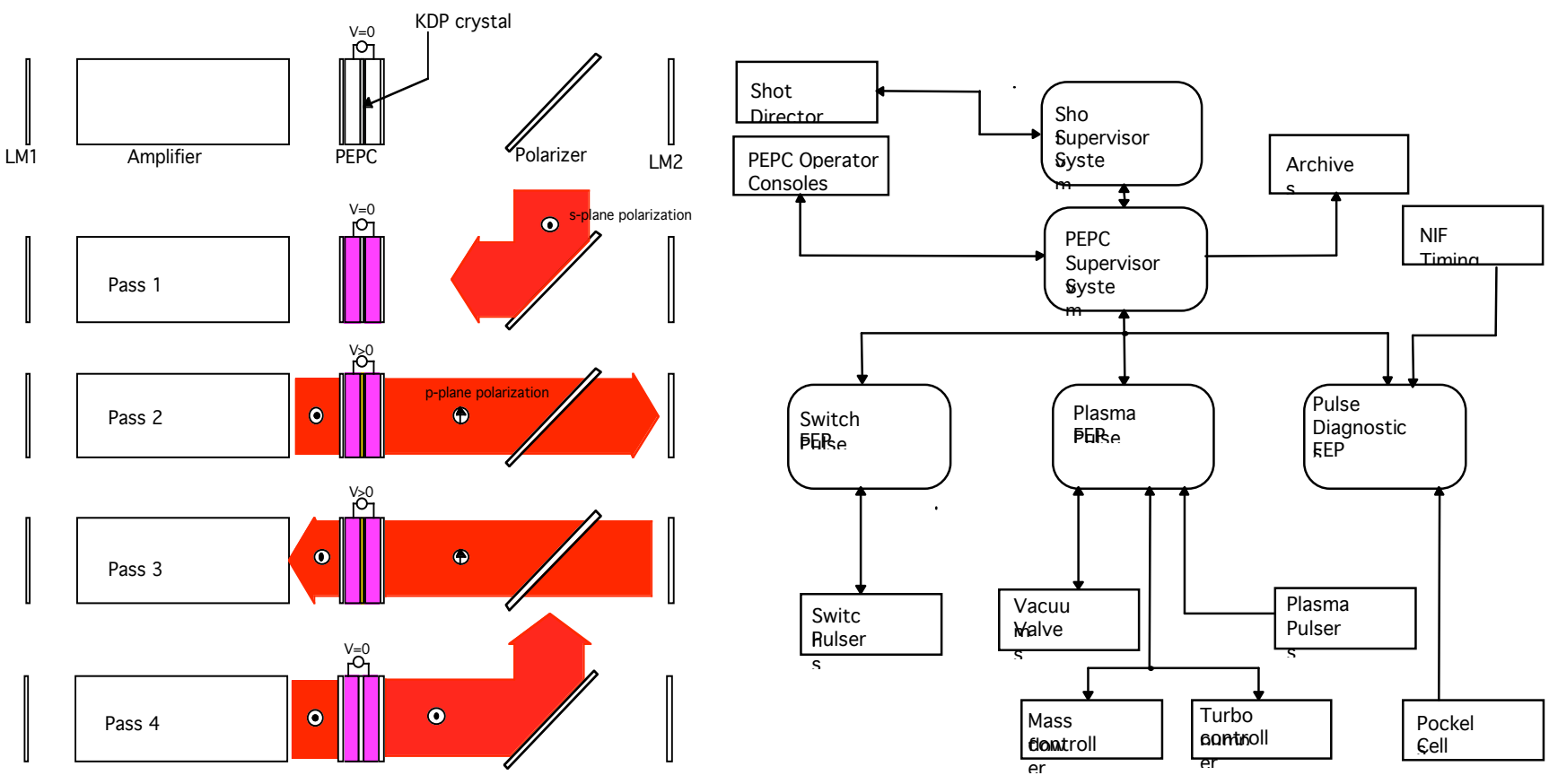

Figure 2. PEPC operation during a laser shot.

\section{Plasma Electrode Pockels Cell Control System}

The function of the PEPC control system is to provide remote (automated) control and monitoring of the PEPC subsystem during NIF operations. The system controls all the components of the PEPC system, including plasma pulsers, switch pulsers, vacuum and gas gauges, valves and turbo pumps.

As with other segments of the NIF control system, the PEPC control system is based on front-end processors (FEPs) that connect upstream to supervisory software modules and downstream to input/output interfaces, as shown in Figure 3. Each cluster of 12 PEPC Line Replaceable Units (LRUs) will require 3 FEPs. Because there are 4 clusters in NIF, the PEPC control system will have a total of 12 FEPs. The 3 FEPs per cluster divide the control duty for the PEPC subsystems. The first FEP controls the switch pulsers. The second FEP controls the plasma-pulser controls, monitors the individual gas and vacuum system on each LRU, and monitors the clusterwide vacuum roughing system. The third FEP controls pulse diagnostics. It contains high-speed waveform digitizers for the acquisition of switch-pulse voltage and plasma-pulse current.

During a NIF shot, the PEPC control system turns on the PEPC system, checks for normal operation of each LRU, triggers the PEPC system every 5 seconds until a NIF shot occurs, and then turns the PEPC system off. The control system determines whether an LRU is firing properly by comparing the switch-pulse voltage and plasma-pulse current waveforms with stored reference waveforms. If an alert is detected the control system notifies both the operator and the automated supervisory system that a PEPC is not working properly.

Figure 3. Plasma Electrode Pockels Cell Computer Control System.

\section{Plasma Electrode Pockels Cells Pulsing Sequence}

PEPC operation is essentially limited to the last five minutes of the shot countdown. In particular, 280 seconds before the main laser is fired, the control system initiates a sequence of events that are detailed below.

During a NIF shot countdown, the PEPC system is commanded to go from its 'Ready' state to its 'Pulsing' state by the NIF supervisory software. The transition involves several clearly defined steps that can be monitored from a combination of Graphical User Interfaces (GUI's). The steps describe are the sequence of commands that are automatically issue by the supervisory software in order to take the PEPC system into 'Pulsing' state:

- Inject Process Gas -Mass Flow Controller onboard the PEPC Line Replaceable Unit injects and regulates $\mathrm{He} / \mathrm{O}_{2}$ into the Plasma cells at 70mTorr.

- Simmer Enabled - Enables the simmer supplies located inside the Plasma Pulse Generator.

- PPG's Pulsing - Begins the charge/discharge cycle of the Plasma Pulse Generators at $5 \mathrm{kV}$ and 2 to $2.2 \mathrm{kA}$ that creates the uniform equipotential, high conductivity plasma electrodes in each of the four cell apertures.

- SPG's Pulsing - Begins the charge/discharge cycle of the $17 \mathrm{kV}$ switch pulse applied to a KDP crystal via the plasma electrodes. Switch pulses are typically 400ns in duration, measured at Full Width Half Maximum. 


\section{1) PEPC Plasma Pulser GUI}

The primary function of the Plasma Pulse Monitoring GUI illustrated in Figure 4 is to give the operator a visual indication of the plasma current waveform being applied to the Line Replaceable Unit (LRU) during operation. This GUI displays each of the four PPG waveforms applied to a given LRU. This GUI not only serves as a diagnostic but it also gives operators an immediate indication of PPG health by comparing the waveforms with reference data.

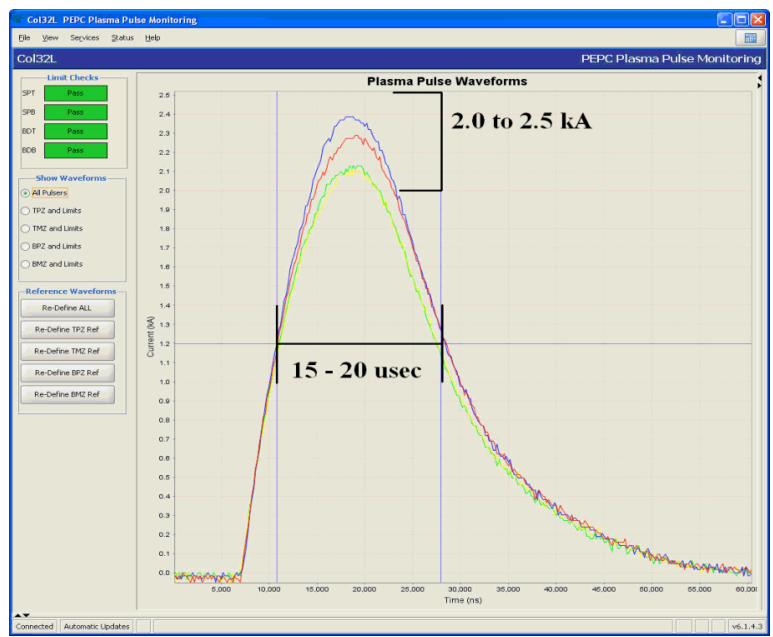

Figure 4. PEPC Plasma Pulse Monitoring GUI.

\section{2) PEPC Switch Pulser GUI}

The primary function of the Switch Pulse Monitoring GUI illustrated in Figure 5 is to give the operator a visual indication of the switch pulse timing, displaying the SPG output current and the cell voltage waveforms. As part of the pre-shot sequence, an auto-timing feature temporally overlays the output currents and aligns them to a timing fiducial.

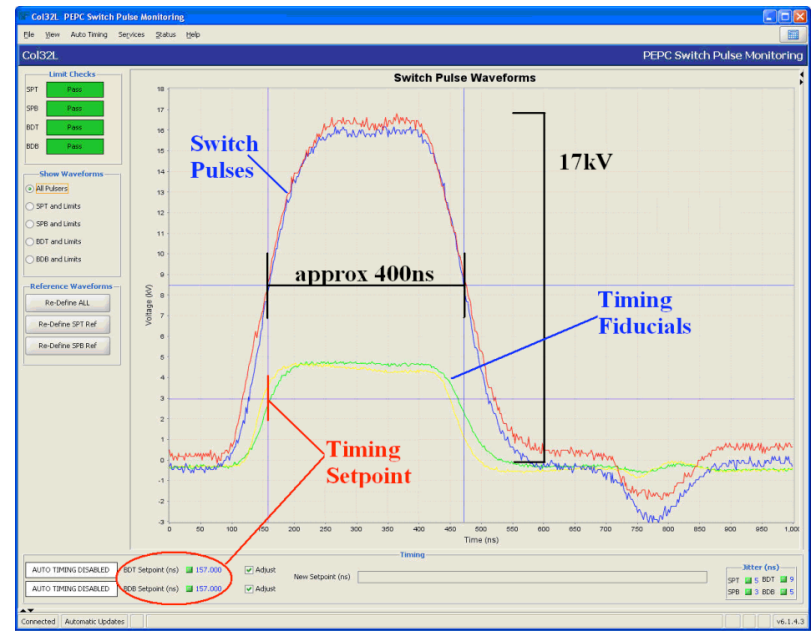

Figure 5. PEPC Switch Pulse Monitoring GUI.

\section{PULSED POWER ASPECTS OF THE PLASMA ELECTORDE POCKELS CELL}

Each Pockels cell is supported by four plasma pulse generators (PPGs) and two switch pulse generators (SPGs). In all, ten independent excitations are required to effect the polarization rotation of the laser light. The PPGs provide the plasma "electrodes" for the KDP crystals. The SPGs impress a uniform electric field on the two pairs of KDP crystals that reside inside the cell.

\section{A. Plasma Pulsed Generators (PPGs)}

The four PPGs provide eight independent excitations to the process gas mixture $\left(99 \% \mathrm{He}, 1 \% \mathrm{O}_{2}\right)$ in order to produce the requisite equipotential surfaces on the face of the KDP crystals. Low voltage, low current simmer supplies residing in the PPGs initiate a plasma in the process gas. This initiation process is aided by a so-called "starter anode" located in closed proximity to the planar magnetron cathodes. The small gap is easily broken down and supplies ions and electrons necessary to create a uniform glow discharge across the full aperture. The simmer supplies operate at $1.3 \mathrm{kV}$. Once the supply detects a breakdown, the supply switches to a current regulated mode to maintain the low energy plasma. The simmer discharge is started $300 \mathrm{~ms}$ prior to the arrival of the main plasma discharge and extends for $100 \mathrm{~ms}$ after the optical pulse has passed. Figure 6 illustrates the simmer discharge that is observed in a Plasma Electrode Pockels Cell during electrical testing.

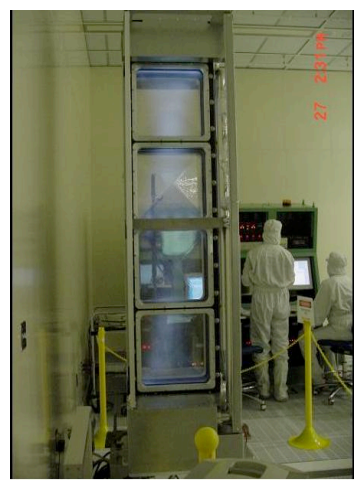

Figure 6. Simmer Discharge in Plasma Electrode Pockels Cell.

Plasma Pulse Generators are also equipped with a capacitive discharge circuit that delivers $60 \mathrm{~J}$ of energy to each plasma channel in the cell. A simplified schematic of the PPG is shown in Figure 7. Light triggered SCRs have recently supplanted thyratrons as the main switch. 


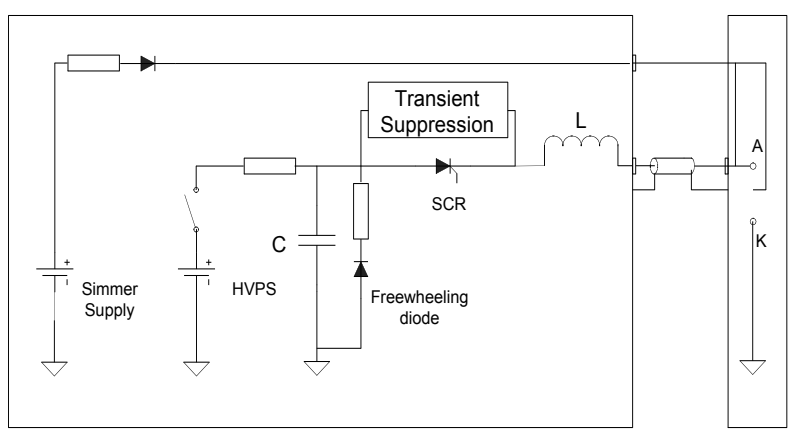

Figure 7. PPG Simplified Schematic.

During the main PPG discharge the electron density in the process gas is increased to greater than $10^{12} \mathrm{~cm}^{-3}$, a conductivity sufficient to produce the desired plasma "electrodes." A fraction of the energy is diverted to a magnetic spreading circuit (utilizing $\mathbf{J} \times \mathbf{B}$ forces to offset plasma pinching) and for biasing the aluminum cell housing near the anode potential to further promote uniform spreading of the plasma throughout the cell aperture. The uniformity of the plasma is evident in Figure 8, captured during the main plasma discharge. [4]

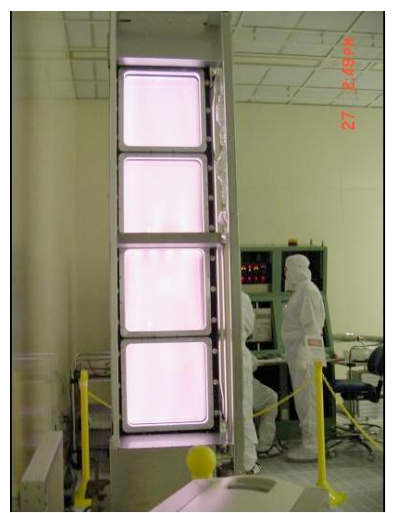

Figure 8. Plasma Pulse Generators Main Discharge.

Installation, deployment and commissioning activities for the Plasma Pulse Generators are (PPGs) currently in progress. In fact, more than 150 of the total 192 PPGs have been installed in the facility. Ninety-six of these devices have been successfully commissioned and are currently supporting 2-shift-per-day shot operations. An additional 48 PPGs are installed in a second laser bay with activation and commissioning exercises proceeding rapidly.

\section{B. Switch Plasma Pulse Generators (SPGs)}

The SPGs provides the necessary bias to the KDP crystals that induces the required birefringence and thus effecting the required $90^{\circ}$ polarization rotation of the light traversing the Pockels cell. Each SPG delivers a $17 \mathrm{kV}$, $20 \mathrm{~J}, 100 \mathrm{~ns}$ risetime rectangular pulse to a load characterized by a capacitance of approximately $6 \mathrm{nF}$, shunted by a series R-L circuit that modified the rise- and falltimes. The maximum operation repetition rate is 1 pulse/ 5 seconds $(0.2 \mathrm{~Hz})$. The SPG consists of a low impedance $(6.25 \Omega)$ pulse forming line (PFL) composed of eight sections of RG-217 configured in parallel, commutated by a thyratron (EEV Model CX-2282). The high voltage pulse propagates through 60 meters of coaxial cable to the Pockels cell. A current transformer monitoring the thyratron current provides timing fiducial for precise overlap of SPG pulses at the cell. A simplified schematic of the SPG is illustrated in Figure 9.

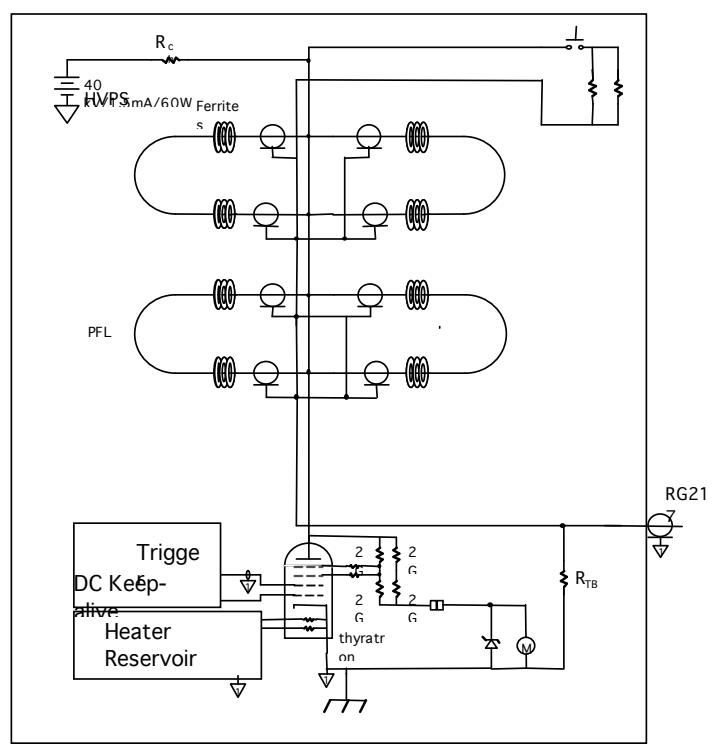

Figure 9. Switch Pulse Generator Simplified Schematic.

Production of the thyratron-switched pulse generator is now complete, with the more than $80 \%$ of the hardware deployed in the facility. Currently, 48 SPGs are supporting shot operations, 32 are in the commissioning phase while 16 are awaiting final testing before being installed.

\section{PLASMA ELECTRODE POCKELS CELL DEPLOYMENT AND OPERATIONS}

Nearly $75 \%$ of the PEPC system supporting hardware has been installed in the National Ignition Facility. The hardware includes 150 Plasma Pulse Generators, 80 Switch Pulse Generators and $100 \%$ of the controls and diagnostics chassis. Operation of the PEPC system is fully automated, with a computer control system and central timing system controlling and monitoring all aspects of pulser and cell performance. On average 20 low and 4 high-energy shots are fired daily during 2 -shift operations. 


\section{FUTURE}

Before the end of summer 2007, 288 pulse generators (96 SPGs and 192 PPGs) will have been deployed in the NIF providing the necessary pulse power to drive all 48 PEPCs. By year's end the entire PEPC system will be operational and ready to support NIF's fusion research.

\section{REFERENCES}

[1] Charles D. Boley and Mark A. Rhodes, "Modeling of Plasma Behavior in a Plasma Electrode Pockels Cell," IEEE Trans. Plasma Sci., Vol. 27, pp. 713-726, June 1999.

[2] Mark A. Rhodes, Scott Fochs, Peter Biltoft, Terry Alger, Bill Funkhouser, C.D. Boley, Craig Ollis, Cal Robb, “4x1 PEPC Operational Prototype," NIF Document 0012961 , August 1998.

[3] P.A. Arnold, NIF College: Plasma Electrode Pockels Cell (PEPC) Overview, NIF Internal Document.

[4] Bill DeHope, Andy Hinz, and Craig Ollis, "Investigation of Magnetic Interactions among NIF PEPCs", NIF Internal Memo, April 5, 2002. 